\title{
Warped Product Pointwise Semi Slant Submanifolds of Sasakian Space Forms and their Applications
}

\author{
Nadia Alluhaibi ${ }^{1}$ and Meraj Ali Khan $\mathbb{D}^{2}$ \\ ${ }^{1}$ Department of Mathematics, Science and Arts College, Rabigh Campus, King Abdulaziz University, Jeddah, Saudi Arabia \\ ${ }^{2}$ Department of Mathematics, University of Tabuk, Saudi Arabia \\ Correspondence should be addressed to Meraj Ali Khan; meraj79@gmail.com
}

Received 20 August 2019; Revised 7 November 2019; Accepted 25 November 2019; Published 17 January 2020

Academic Editor: Dimitrios Tsimpis

Copyright (c) 2020 Nadia Alluhaibi and Meraj Ali Khan. This is an open access article distributed under the Creative Commons Attribution License, which permits unrestricted use, distribution, and reproduction in any medium, provided the original work is properly cited.

In this study, we attain some existence characterizations for warped product pointwise semi slant submanifolds in the setting of Sasakian space forms. Moreover, we investigate the estimation for the squared norm of the second fundamental form and further discuss the case of equality. By the application of attained estimation, we obtain some classifications of these warped product submanifolds in terms of Ricci soliton and Ricci curvature. Further, the formula for Dirichlet energy of involved warping function is derived. A nontrivial example of such warped product submanifolds is also constructed. Throughout the paper, we will use the following acronyms: "WP" for warped product, "WF" for warping function, "AC" for almost contact, and "WP-PSS" for the warped product pointwise semi slant.

\section{Introduction}

In the field of geometry, an increased interest is observed for the exploration of the warped product manifolds due to its applicability in the theory of relativity and physics [1]. Earlier researchers have shown that warped products facilitate the computation of the Einstein field equations [1]. In addition, the concept of warped product manifolds is used in the notion of modelling of space-time near black holes [2], such as Schwarzschild space-time $P \times{ }_{r} S^{2}$, where $r>0$, the base is a half plane $P=R \times R^{+}$, and the unit sphere $S^{2}$ is the fibre. At some instances, Schwarzschild space-time also acts similar to the black hole. The Robertson-Walker model is an example of a warped product which is considered as a cosmological model adopted for the space-time modelling of the universe [3].

Identifying Dirichlet energy of smooth functions is considered as an integral part in the field of physics and engineering. Moreover, Dirichlet energy is found to be an analog of kinetic energy. Let $\tau$ be any real valued smooth function on a compact manifold $M$, then the Dirichlet energy of $\tau$ is defined by

$$
E(\tau)=\frac{1}{2} \int_{M}\|\nabla \tau\|^{2} d V
$$

where $\nabla \tau$ is the gradient of $\tau$ and $d V$ is the volume element. It is clear that $E(\tau) \geq 0$. It is well recognized that a product manifold cannot be used for the representation of nonzero (constant) curvature manifolds. Therefore, the concept of the warped product manifold emerged due to the nonexistence of negative curvature for the Riemannian product of manifolds. Therefore, Bishop and O'Neill [4] initiated the concept of warped product manifolds for devising the class of manifolds for the negative or nonpositive curvature. A warped product manifold (see definition in Section 2) is a generality of a product manifold. As the warping function of warped product manifolds is a positive valued smooth function, the focus of the present study is on examining the Dirichlet energy of such functions. 
The study of Bishop and O'Neill [4] has enlightened a few intrinsic properties of the warped product manifolds. Initial extrinsic studies of the warped product manifold in the almost complex setting were performed by Chen $[5,6]$ while obtaining some results of existence for CRsubmanifold as CR-warped product submanifold in Kaehler manifolds. Furthermore, contact CR-warped product submanifolds were investigated in the study of Hasegawa and Mihai [7] in the almost contact settings. Warped product manifolds are also investigated in the contact setting by many other geometers which have attained various existence results [8-11].

Warped product pointwise semi slant submanifold is another generalized class of warped product semi slant submanifolds and contact CR-warped product submanifolds. In [12], Park studied such warped product submanifolds. After that, Mihai and Uddin extended this study in Sasakian manifolds and acquired some optimal inequalities related to warping function and second fundamental form. Warped product pointwise semi slant submanifolds for almost contact and almost complex manifolds were explored in [13-15].

On the other hand, the gradient Ricci soliton is extensively investigated in physics, theory of relativity, and differential geometry. The classification results related to Ricci soliton and gradient Ricci solitons with the warped product structure have been established in [16-19]. Moreover, the Ricci curvature has a significant nature in Riemannian geometry; for example, Ricci flat is used to solve the Einstein field equation on a Riemannian manifold in which the cosmological constant vanishes. More clearly, in the general relativity theory, the Ricci tensor is correlated with Einstein's field equation to study the material contents of the universe. So, in comparison with Riemannian curvature, the Ricci curvature is more significant in the theory of relativity and physics.

In this study, warped product pointwise semi slant submanifolds are studied in the setting of Sasakian space forms and some associated inequalities are attained. The study also provides estimates for the squared norm of the second fundamental form concerning the slant function and warping function and then addresses the case of equality. Some applications of the case of equality and the derived inequality are explored. More precisely, Dirichlet energy of the warping functions is computed via the obtained inequality. Finally, a classification of the WP-PSS submanifolds admitting the gradient Ricci soliton, in respect of Ricci curvature and second fundamental form, is obtained, and some existence results are also established.

The study consists of five sections in which the second section is dedicated to pave the way for the other sections by providing the fundamental definition, formulas, and preliminary outcomes needed for the study. Section 3 investigates the WP-PSS submanifold existence in Sasakian space forms and provides the proof of the main findings of the study. In addition, it gives a nontrivial example for the WP-PSS submanifolds in the setting of a Sasakian manifold. The formulas for Dirichlet energy of warping function is attained in Section 4 by using the derived inequality. The paper is concluded in Section 5, followed by its bibliography.

\section{Preliminaries}

A $C^{\infty}$ - manifold $\bar{M}$ of dimension $(2 m+1)$ is said to admit an $A C$ structure if on $\bar{M} \exists$ a tensor field $\Psi$ of type $(1,1)$, a vector field $\eta$, and a 1 -form $\zeta$ fulfilling [20]

$$
\Psi^{2}=-I+\zeta \otimes \eta, \quad \Psi \eta=0, \zeta \circ \Psi=0, \zeta(\eta)=1 .
$$

The manifold $\bar{M}$ with the structure $(\Psi, \eta, \zeta)$ is called $A C$ metric manifold. On an AC metric manifold $\bar{M}, \exists$ a Riemannian metric $g$ satisfying

$$
\zeta(Y)=g(Y, \eta), \quad g(\Psi Y, \Psi V)=g(Y, V)-\zeta(Y) \zeta(V)
$$

for all $Y, V \in T \bar{M}$, where $T \bar{M}$ is the tangent bundle of $\bar{M}$.

An AC metric structure $(\Psi, \eta, \zeta, g)$ is referred to as Sasakian manifold if it fulfills the following relation [20]:

$$
\left(\bar{\nabla}_{Y} \Psi\right) V=g(Y, V) \eta-\zeta(V) Y
$$

for any $Y, V \in T \bar{M}$, where $\bar{\nabla}$ denotes the Riemannian connection of the metric $g$. Then, we have

$$
\bar{\nabla}_{Y} \eta=-\Psi Y
$$

A Sasakian space form $\bar{M}(c)$ [20] is a Sasakian manifold with constant $\Psi$-holomorphic sectional curvature $c$. Moreover, the following formula gives the curvature tensor $\bar{R}$ of Sasakian space form, $\bar{M}(c)$ :

$$
\begin{aligned}
\bar{R}\left(Y_{1}, Y_{2}\right) V= & \frac{c-3}{4}\left\{g\left(Y_{2}, V\right) Y_{1}-g\left(Y_{1}, V\right) Y_{2}\right\} \\
& +\frac{c-1}{4}\left\{g\left(Y_{1}, \Psi V\right) \Psi Y_{2}-g\left(Y_{2}, \Psi V\right) \Psi Y_{1}\right. \\
& +2 g\left(Y_{1}, \Psi Y_{2}\right) \Psi V+\zeta\left(Y_{1}\right) \zeta(V) Y_{2} \\
& -\zeta\left(Y_{2}\right) \zeta(V) Y_{1}+g\left(Y_{1}, V\right) \zeta\left(Y_{2}\right) \eta \\
& \left.-g\left(Y_{2}, V\right) \zeta\left(Y_{1}\right) \eta\right\}
\end{aligned}
$$

$\forall$ vector fields $Y_{1}, Y_{2}, V$ on $\bar{M}$.

Let $M$ be a submanifold of an AC-metric manifold $\bar{M}$ with induced metric $g$, and the vector field $\eta$ is tangential to $M$. The Riemannian connection $\bar{\nabla}$ of $\bar{M}$ induces canonically the connections $\nabla^{\perp}$ and $\nabla$ on the normal bundle $T^{\perp} M$ and the tangent bundle $T M$ of $M$, resp. Then, the Weingarten and Gauss formulas are as follows:

$$
\begin{gathered}
\bar{\nabla}_{Y_{1}} N=-A_{N} Y_{1}+\nabla_{Y_{1}}^{\perp} N, \\
\bar{\nabla}_{Y_{1}} Y_{2}=\nabla_{Y_{1}} Y_{2}+\sigma\left(Y_{1}, Y_{2}\right),
\end{gathered}
$$

$\forall Y_{1}, Y_{2} \in T M$ and $N \in T^{\perp} M$, where $A_{N}$ and $\sigma$ are the shape operator and the second fundamental form, resp. For the immersion of $M$ into $\bar{M}, A_{N}$ and $\sigma$ verify the relation

$$
g\left(\sigma\left(Y_{1}, Y_{2}\right), N\right)=g\left(A_{N} Y_{1}, Y_{2}\right)
$$


If $M$ is a submanifold of a Riemmanian manifold $\bar{M}$, then the equation of Codazzi is as follows:

$$
\begin{aligned}
\left(\bar{R}\left(Y_{1}, Y_{2}\right) V\right)^{\perp}= & \nabla_{Y_{1}}^{\perp} \sigma\left(Y_{2}, V\right)-\nabla_{Y_{2}}^{\perp} \sigma\left(Y_{1}, V\right) \\
& +\sigma\left(\nabla_{Y_{2}} Y_{1}, V\right)-\sigma\left(\nabla_{Y_{1}} Y_{2}, V\right) \\
& +\sigma\left(Y_{1}, \nabla_{Y_{2}} V\right)-\sigma\left(Y_{2}, \nabla_{Y_{1}} V\right)
\end{aligned}
$$

where $\left(\bar{R}\left(Y_{1}, Y_{2}\right) V\right)^{\perp}$ denotes the normal component of the curvature tensor $\bar{R}\left(Y_{1}, Y_{2}\right) V$.

If $P Y$ and $F Y$ denote the tangential and normal component of $\Psi Y$, respectively, then

$$
\Psi Y=P Y+F Y
$$

$\forall Y \in T M$. Similarly, for any normal vector field $N \in T^{\perp} M$,

$$
\Psi N=t N+f N
$$

Thus, $F$ (resp., $t$ ) is a normal (resp., tangential) valued 1form on $T M$ (resp. $T^{\perp} M$ ) and $P$ (resp., $f$ ) is 1-1 tensor field on $T M$ (resp., $T^{\perp} M$ ). The covariant derivatives of $\Psi, F$, and $P$ are given by

$$
\begin{aligned}
& \left(\bar{\nabla}_{Y_{1}} \Psi\right) Y_{2}=\nabla_{Y_{1}} \Psi Y_{2}-\Psi \nabla_{Y_{1}} Y_{2}, \\
& \left(\bar{\nabla}_{Y_{1}} F\right) Y_{2}=\nabla_{Y_{1}}^{\perp} F Y_{2}-F \nabla_{Y_{1}} Y_{2}, \\
& \left(\bar{\nabla}_{Y_{1}} P\right) Y_{2}=\nabla_{Y_{1}} P Y_{2}-P \nabla_{Y_{1}} Y_{2} .
\end{aligned}
$$

From equations (4), (8), (7), (11), and (12), we have

$$
\left(\bar{\nabla}_{Y_{1}} P\right) Y_{2}=A_{F Y_{2}} Y_{1}+t \sigma\left(Y_{1}, Y_{2}\right)-g\left(Y_{1}, P Y_{2}\right) \eta-\zeta\left(Y_{2}\right) P Y_{1}
$$

$\left(\bar{\nabla}_{Y_{1}} F\right) Y_{2}=f \sigma\left(Y_{1}, Y_{2}\right)-\sigma\left(Y_{1}, P Y_{2}\right)-\zeta\left(Y_{2}\right) F Y_{1}$

Let $\left\{e_{1}, e_{2}, \cdots, e_{m}\right\}$ be a local orthonormal basis of vector fields on $m$-dimensional submanifold $M$. Hence, the mean curvature vector $H$ of $M$ is obtained from the formula

$$
H=\frac{1}{m} \sum_{s=1}^{m} \sigma\left(e_{s}, e_{s}\right),
$$

and the squared norm of the second fundamental form $\sigma$ is given by

$$
\|\sigma\|^{2}=\sum_{r, s=1}^{m} g\left(\sigma\left(e_{r}, e_{s}\right), \sigma\left(e_{r}, e_{s}\right)\right) .
$$

A submanifold $M$ of $\bar{M}$ is called totally umbilical if $\sigma$ $\left(Y_{1}, Y_{2}\right)=g\left(Y_{1}, Y_{2}\right) H$, and $M$ is defined as totally geodesic if $\sigma\left(Y_{1}, Y_{2}\right)=0, \forall Y_{1}, Y_{2} \in T M$.

Definition 1 (see [21]). A submanifold $M$ of an AC metric manifold $\bar{M}$ is slant if the angle between $Y$ and $\Psi Y$ is con- stant for any $y \in M$ and $Y \in T_{y} M-\langle\eta\rangle$. Then the constant angle $\theta$ s.t. $0 \leq \theta \leq \pi / 2$ is called slant angle of $M$ in $\bar{M}$.

Definition 2 (see [21]). If $\theta=0$, the submanifold $M$ is invariant submanifold and is anti-invariant submanifold if $\theta=\pi / 2$. If $\theta$ is neither 0 nor $\pi / 2, M$ is proper slant submanifold.

Etayo [22] introduced the concept of pointwise slant submanifolds as a natural generality of the slant submanifolds of almost Hermitian manifolds. Later, Chen and Garay [23] investigated pointwise slant submanifolds in the setting of almost Hermitian manifolds and attained some primary results. A step forward, Park [12] extended the notion of pointwise slant submanifolds in the setting of AC-metric manifolds. Recently, Uddin and Al-Khalidi [24] modified the definition of pointwise slant submanifolds for ACmetric manifolds. More precisely, a submanifold $M$ of an AC-metric manifold $\bar{M}$ is referred to as poinwise slant submanifold if $\forall Y \in T_{y} M$ such that $\eta \in T M$ the angle $\theta(Y)$ between $\Psi Y$ and $T_{y} M-\{0\}$ is independent of the choice of nonzero vector field $Y \in T_{p} M-\{0\}$. Hence, $\theta$ is dealt with as the function on $M$, which is known as a slant function of the pointwise slant function. Now, we attain the following characterizing theorem.

Theorem 3 (see [24]). Suppose that $M$ is a submanifold of an AC-metric manifold $\bar{M}$ such that $\eta \in T M$. Then, $M$ is pointwise slant if and only if

$$
P^{2}=\cos ^{2} \theta(-I+\zeta \otimes \eta)
$$

where $\theta: T M \longrightarrow R$.

Consequently, we have

$$
\begin{gathered}
g\left(P Y_{1}, P Y_{2}\right)=\cos ^{2} \theta\left[g\left(Y_{1}, Y_{2}\right)-\zeta\left(Y_{1}\right) \zeta\left(Y_{2}\right)\right], \\
g\left(F Y_{1}, F Y_{2}\right)=\sin ^{2} \theta\left[g\left(Y_{1}, Y_{2}\right)-\zeta\left(Y_{1}\right) \zeta\left(Y_{2}\right)\right], \\
\forall Y_{1}, Y_{2} \in T M .
\end{gathered}
$$

Recently, Mihai and Uddin [15] defined and studied pointwise semi slant submanifold of Sasakian manifolds.

Definition 4. A submanifold $M$ of an AC-metric manifold $\bar{M}$ is referred to as a pointwise semi slant submanifold if $\exists$ two orthogonal complementary distributions $D$ and $D^{\theta}$ on $M$ such that

(i) $T M=D \oplus D^{\theta} \oplus\langle\eta\rangle$

(ii) $D$ is invariant

(iii) $D^{\theta}$ is pointwise slant with a slant function $\theta$

The WP manifolds are the generalized form of the product manifolds and are defined as follows. 
Let $\left(C_{1}, g_{C_{1}}\right)$ and $\left(C_{2}, g_{C_{2}}\right)$ be two Riemannian manifolds with the Riemannian metrics $g_{C_{1}}$ and $g_{C_{2}}$, resp., and $\Phi$ be a positive differentiable map on $C_{1}$. Let $\pi_{C_{1}}: C_{1} \times C_{2} \longrightarrow C_{1}$, $\pi_{C_{2}}: C_{1} \times C_{2} \longrightarrow C_{2}$ be the projection maps given by $\pi_{C_{1}}$ $\left(c_{1}, c_{2}\right)=c_{1}$ and $\pi_{C_{2}}\left(c_{1}, c_{2}\right)=c_{2}, \forall\left(c_{1}, c_{2}\right) \in C_{1} \times C_{2}$. The WP $M=C_{1} \times{ }_{\Phi} C_{2}$ [4] is the manifold $C_{1} \times C_{2}$ endowed with the Riemannian structure s.t.

$$
\begin{aligned}
g\left(Y_{1}, Y_{2}\right)= & g_{C_{1}}\left(\left(\pi_{C_{1}}\right)_{*} Y_{1},\left(\pi_{C_{1}}\right)_{*} Y_{2}\right) \\
& +\left(\Phi \circ \pi_{C_{1}}\right)^{2} g_{C_{2}}\left(\left(\pi_{C_{2}}\right)_{*} Y_{1},\left(\pi_{C_{2}}\right)_{*} Y_{2}\right),
\end{aligned}
$$

for all $Y_{1}, Y_{2} \in T M$, where $*$ denotes the tangential map (the differential on the tangent bundle). Here, $\Phi$ is known as the WF of the WP manifold. $M$ is a trivial WP if the $\mathrm{WF}$ is constant.

Let $Y_{1}$ be a vector field on $C_{1}$ and $Y_{2}$ be a vector field on $C_{2}$, then we induce from Lemma 7.3 of [3]:

$$
\nabla_{Y_{1}} Y_{2}=\nabla_{Y_{2}} Y_{1}=\left(\frac{Y_{1} \Phi}{\Phi}\right) Y_{2}
$$

where $\nabla$ is the Levi-Civita connection on $M$. For $M=C_{1} \times{ }_{\Phi} C_{2}$, it can be seen that

$$
\nabla_{Y_{1}} Y_{2}=\nabla_{Y_{2}} Y_{1}=\left(Y_{1} \ln \Phi\right) Y_{2},
$$

for $Y_{1} \in T C_{1}$ and $Y_{2} \in T C_{2}$. The gradient of $\Phi$ is denoted by $\nabla \Phi$ and is defined as

$$
g(\nabla \Phi, Y)=Y \Phi, \quad \forall Y \in T M
$$

Let $M$ be a Riemannian manifold $M$ of dimension $m$ with $\left\{e_{1}, \cdots, e_{m}\right\}$ as an orthogonal basis of $T M$ and $g$ a Riemannian metric of $M$. Then, as a result of (27), we get

$$
\|\nabla \Phi\|^{2}=\sum_{s=1}^{m}\left(e_{s}(\Phi)\right)^{2}
$$

The Laplacian of a smooth function $\Phi$ on a Riemannian manifold is defined by the following equation:

$$
\Delta \Phi=\sum_{s=1}^{m}\left\{\left(\nabla_{e_{s}} e_{s}\right) \Phi-e_{s} e_{s} \Phi\right\}=-\sum_{i=1}^{m} g\left(\nabla_{e_{i}} \operatorname{grad} \Phi, e_{i}\right)
$$

For a smooth function $\Phi$ on $M$, the Hessian of $\Phi$ is defined as

$$
H^{\Phi}(U, V)=U V \Phi-\left(\nabla_{U} V\right) \Phi=g\left(\nabla_{U} \operatorname{grad} \Phi, V\right)
$$

for any $U, V \in T M$.
The Hessian tensor for a differential function $\Phi$ is a symmetric covariant tensor of rank 2 and is defined as

$$
\Delta \Phi=-\operatorname{trace} H^{\Phi} .
$$

Throughout the study, we used the following relation between the Hessian tensor and the Laplacian of a smooth function $\Phi$ on a Riemannian manifold:

$$
\text { Hess }^{\Phi}=-\Delta \Phi .
$$

Hopf s Lemma [25]. If $M$ is an $m$-dimensional connected compact Riemannian manifold and $\Phi$ is a differentiable function on $M$ s.t. $\Delta \Phi \leq 0$ everywhere on $M$ (or $\Delta \Phi \geq 0$ everywhere on $M$ ), then $\Phi$ is a constant function.

For a compact orientable Riemannian manifold $M$ with or without boundary and as a consequence of the integration theory on manifolds, we have [26]

$$
\int_{M} \Delta \Phi d V=0
$$

where $\Phi$ is a function on $M$ and $d V$ is the volume element of $M$.

The Ricci soliton idea was given by Hamilton [26]. It is regarded as the natural generality of Einstein metrics, and it is the self-similar solution of the Ricci flow $(\partial / \partial t) g(t)=-2$ $\operatorname{Ric}(t)$. If $\exists$ a smooth vector field $Y$ such that the Ricci tensor meets the following condition:

$$
\operatorname{Ric}+\frac{1}{2} \mathscr{L}_{Y} g=\alpha,
$$

for any constant $\alpha$, where $\mathscr{L}_{Y}$ is the Lie derivative, then the metric $g$ on a complete Riemannian manifold $\bar{M}$ is named as Ricci soliton. If $\alpha<0, \alpha=0$, and $\alpha>0$, then the Ricci soliton is called expanding, steady, and shrinking, respectively. If we specify $Y=\nabla \Phi$ for a smooth function $\Phi$ on $\bar{M}$, then $g$ admits gradient Ricci soliton with the potential function $\Phi$. For this case, (34) takes the form

$$
\operatorname{Ric}+\nabla^{2} \Phi=\alpha g .
$$

Since the Laplacian $\Delta$ and the gradient $\nabla^{2}$ are related as $\Delta=\nabla^{2}$, in terms of Hessian, (35) can be expressed as

$$
\text { Ric }=\alpha g+\text { Hess }^{\psi} .
$$

Note 1. $(M, g, \nabla \Phi, \alpha)$ is an Einstein manifold if the potential function $\Phi$ is constant on a gradient Ricci soliton.

\section{Warped Product Pointwise Semi Slant Submanifolds}

In [12], Park proved that the WP-PSS submanifolds of the type $N_{T} \times{ }_{\Phi} N_{\theta}$ of the contact metric manifolds exist and achieved the result of the following lemma. 
Lemma 5. Suppose that $M=N_{T} \times{ }_{\Phi} N_{\theta}$ is a WP-PSS of a Sasakian manifold $\bar{M}$ such that $\eta \in T N_{T}$, where $N_{T}$ and $N_{\theta}$ are invariant and pointwise slant submanifolds of $\bar{M}$, respectively. Then, we have

$$
\begin{aligned}
g(\sigma(Y, W), F P Z)= & \cos ^{2} \theta Y \ln \Phi g(W, Z) \\
& -\Psi Y \ln \Phi g(W, P Z)-\zeta(Y) g(W, P Z),
\end{aligned}
$$

$\forall Y \in T N_{T}$ and $W, Z \in T N_{\theta}$.

In particular, if we replace $Z$ by $P W$ in the above equation, then one can conclude the following:

$$
g(\sigma(Y, W), F W)=-\Psi Y \ln \Phi\|W\|^{2}-\zeta(Y)\|W\|^{2}
$$

Now, let $M=N_{T} \times{ }_{\Phi} N_{\theta}$ be a WP-PSS of a Sasakian manifold $\bar{M}$ and we consider $\eta \in T N_{T}$. If $D$ is invariant distribution and $D^{\theta}$ is proper pointwise slant distribution with $\theta$ as a slant function, then $T M$ and $T^{\perp} M$ are decomposed (resp.) as follows:

$$
\begin{aligned}
T M & =D \oplus D^{\theta} \oplus\langle\eta\rangle, \\
T^{\perp} M & =F D^{\theta} \oplus \mu,
\end{aligned}
$$

where $\mu$ is the orthogonal complementary distribution of $F D^{\theta}$ in $T^{\perp} M$. One can easily check that $\mu$ is an invariant subbundle of $T^{\perp} M$ regarding $\Psi$.

In view of the above direct decomposition, $\sigma$ can be written as

$$
\sigma\left(U_{1}, U_{2}\right)=\sigma_{F D^{9}}\left(U_{1}, U_{2}\right)+\sigma_{\mu}\left(U_{1}, U_{2}\right)
$$

for $U_{1}, U_{2} \in T M$, where $\sigma_{F D^{\theta}}\left(U_{1}, U_{2}\right)$ and $\sigma_{\mu}\left(U_{1}, U_{2}\right)$ are the components of $\sigma\left(U_{1}, U_{2}\right)$ in the normal subbundles $F D^{\theta}$ and $\mu$, respectively. Moreover, if $\left\{V_{1}, V_{2}, \cdots, V_{q}\right\}$ is a local orthonormal basis of vector fields of $D^{\theta}$, we have

$$
\sigma_{F D^{\theta}}\left(U_{1}, U_{2}\right)=\sum_{r=1}^{q} \sigma^{r}\left(U_{1}, U_{2}\right) F V_{r}
$$

where

$$
\sigma^{r}(U, V)=\csc ^{2} \theta g\left(\sigma(U, V), F V_{r}\right) .
$$

To ensure the existence, we construct an example of a WP-PSS of the type $M=N_{T} \times{ }_{\Phi} N_{\theta}$ in the Sasakian manifold with $\eta$ tangent to $N_{T}$.
Example 1. We know that $\left(R^{2 m+1}, \Psi_{0}, \zeta, \eta, g\right)$ denotes a Sasakian manifold with the structure as follows:

$$
\zeta=\frac{1}{2}\left(d z-\sum_{i=1}^{m} y^{i} d x^{i}\right)
$$$$
\eta=2 \frac{\partial}{\partial z}
$$$$
g=\zeta \otimes \zeta+\frac{1}{4}\left(\sum_{i=1}^{m}\left(d x^{i} \otimes d x^{i}+d y^{i} \otimes d y^{i}\right)\right),
$$

$$
\begin{aligned}
& \Psi_{0}\left(\sum_{i=1}^{m}\left(X_{i} \frac{\partial}{\partial x^{i}}+Y_{i} \frac{\partial}{\partial y^{i}}+Z \frac{\partial}{\partial z}\right)\right) \\
& =\sum_{i=1}^{m}\left(Y_{i} \frac{\partial}{\partial x^{i}}-X_{i} \frac{\partial}{\partial y^{i}}\right)+\sum_{i=1}^{m} Y_{i} y^{i} \frac{\partial}{\partial z},
\end{aligned}
$$

where $\left(x^{i}, y^{i}, z\right), i=1, \cdots, m$, are the Cartesian coordinates. It is well known that $\left\{2\left(\partial /\left(\partial y^{i}\right)\right), 2\left(\left(\partial /\left(\partial x^{i}\right)\right)+y^{i}(\partial / \partial z)\right), \eta\right\}$ is an orthonormal basis of $T R^{2 m+1}$.

Now, consider the 9-dimensional Sasakian manifold $\left(R^{9}\right.$, $\left.\Psi_{0}, \zeta, \eta, g\right)$ and a submanifold isometrically immersed in $R^{9}$ as follows:

$$
M=\left\{2\left(u, 0, w e^{t}, 0, v, s e^{t} \cos \theta, s e^{t} \sin \theta, 0, f\right) \in R^{9}\right\},
$$

and consider a basis $\left\{e_{1}, e_{2}, e_{3}, e_{4}, e_{5}\right\}$ of orthogonal vector fields on $M$ as

$$
\begin{aligned}
& e_{1}=2\left(\frac{\partial}{\partial x^{1}}+y^{1} \frac{\partial}{\partial z}\right), \\
& e_{2}=2 \frac{\partial}{\partial y^{1}} \\
& e_{3}=2 e^{t} \frac{\partial}{\partial x^{3}}, \\
& e_{4}=2 e^{t} \cos \theta \frac{\partial}{\partial y^{3}}+2 e^{t} \sin \theta \frac{\partial}{\partial y^{4}}, \\
& e_{5}=2 \frac{\partial}{\partial z}=\eta .
\end{aligned}
$$

Hence, it can be seen that $D=\operatorname{span}\left\{e_{1}, e_{2}, e_{5}\right\}$ and $D^{\theta}=$ $\operatorname{span}\left\{e_{3}, e_{4}\right\}$ define the invariant and pointwise slant distributions with $\theta \in(0, \pi / 2)$ as a slant function on the Sasakian manifold $R^{9}$. If $N_{T}$ and $N_{\theta}$ stand for the integral manifold of $D$ and $D^{\theta}$, respectively, the metric $g$ on $M$ is defined by

$$
g=g_{N_{T}}+e^{2 t} g_{N_{\theta}}
$$

Then, $M=N_{T} \times{ }_{\Phi} N_{\theta}$ is a WP-PSS with the $\Phi(t)=e^{t}$.

In this section, for convention, we denote by $Y, X \in T N_{T}$ and $V, U \in T N_{\theta}$ as the vector fields of respective tangent bundles of $N_{T}$ and $N_{\theta}$. At first, some initial results need to be proved. 
Lemma 6. Suppose that $N_{T} \times{ }_{\Phi} N_{\theta}$ is a WP-PSS of a Sasakian manifold $\bar{M}$. Then, we have

(i) $\eta \ln \Phi=0$

(ii) $g(\sigma(\Psi Y, V), F V)=Y \ln \Phi\|V\|^{2}$

(iii) $g(\sigma(\Psi Y, V), \Psi \sigma(Y, V))=\left\|\sigma_{\mu}(Y, V)\right\|^{2}+\cos ^{2} \theta$ $(Y \ln \Phi)^{2}\|V\|^{2}$ of $\sigma$.

$\forall Y \in T N_{T}$ and $V \in T N_{\theta}$, where $\sigma_{\mu}$ is the $\mu$ component

Proof. From (5), (8), and (26), it is easy to see that $\eta \ln \Phi=0$. Moreover, replacing $Y$ by $\Psi Y$ in (38), using (2) and part (i), we get part (ii). To prove part (iii), on making use of (8) and (4), we get

$$
\sigma(\Psi Y, V)=-\zeta(U) V+\Psi \sigma(Y, V)+\Psi \nabla_{V} Y-\nabla_{V} \Psi Y
$$

By utilizing (26), the form of the above equation can be changed to as follows:

$\sigma(\Psi Y, V)=-\zeta(Y) V+\Psi \sigma(Y, V)+Y \ln \Phi \Psi V-\Psi Y \ln \Phi V$

By comparing the normal parts, we get

$$
\sigma(\Psi Y, V)=\Psi \sigma_{\mu}(Y, V)+Y \ln \Phi F V
$$

taking the inner product with $\Psi \sigma(Y, V)$, we obtain

$$
\begin{aligned}
& g(\sigma(\Psi Y, V), \Psi \sigma(Y, V)) \\
& \quad=\left\|\sigma_{\mu}(Y, V)\right\|^{2}+Y \ln \Phi g(\Psi \sigma(Y, V), F V) .
\end{aligned}
$$

Calculating the last term of the above equation by using (8), (4), and (22) as follows:

$$
g(\Psi \sigma(Y, V), F V)=g(\sigma(\Psi Y, V), F V)-\sin ^{2} \theta Y \ln \Phi\|V\|^{2}
$$

Utilizing part (ii), we get

$$
g(\Psi \sigma(Y, V), F V)=\cos ^{2} \theta Y \ln \Phi\|V\|^{2}
$$

using (50), we obtain the required result.

Lemma 7. Suppose that $M=N_{T} \times{ }_{\Phi} N_{\theta}$ is a WP-PSS submanifold of a Sasakian manifold $\bar{M}$. Then,

$g(\sigma(Y, P V), F V)=-g(\sigma(Y, V), F P V)=-\cos ^{2} \theta Y \ln \Phi\|V\|^{2}$,

for all $Y \in T N_{T}, V \in T N_{\theta}$.
Proof. From part (ii) of Lemma 6, the following is attained:

$$
g(\sigma(P Y, V), F U)+g(\sigma(P Y, U), F V)=2 Y \ln \Phi g(V, U)
$$

$\forall Y \in T N_{T}$ and $V, U \in T N_{\theta}$. Replacing $U$ by $P V \in D^{\theta}$ and using the fact that $V$ and $P V$ are perpendicular, the following is obtained:

$$
g(\sigma(Y, P V), F V)=-g(\sigma(Y, V), F P V) .
$$

By using (15), (16), and (26), we get

$$
P Y \ln \Phi V-Y \ln \Phi P V=t \sigma(Y, V)-\zeta(Y) V
$$

Now, taking the inner product with $U \in T N_{\theta}$ in the above equation, we have

$$
\begin{aligned}
P Y \ln \Phi g(V, U) & -Y \ln \Phi g(P V, U)=-g(\sigma(Y, V), F U) \\
& -\zeta(X) g(V, U) .
\end{aligned}
$$

Interchanging $V$ and $U$ and subtracting the resultant from equation (57) lead to

$$
-g(\sigma(Y, V), F U)+g(\sigma(Y, U), F V)=2 Y \ln \Phi g(V, P U)
$$

In particular, replacing $U$ by $P V \in D^{\theta}$, we get

$g(\sigma(Y, V), F P V)-g(\sigma(Y, P V), F V)=-2 \cos ^{2} \theta Y \ln \Phi\|V\|^{2}$

Using (55) yields

$g(\sigma(Y, P V), F V)=-g(\sigma(Y, V), F P V)=-\cos ^{2} \theta Y \ln \Phi\|V\|^{2}$

Lemma 8. On a WP-PSS submanifold $M=N_{T} \times{ }_{\Phi} N_{\theta}$ of a Sasakian manifold $\bar{M}$, we obtain

$$
\begin{aligned}
\sum_{i=1}^{q} & {\left[\sum_{j, k=1}^{2 p} g\left(\sigma\left(\Psi e_{i}, e^{k}\right), F e^{j}\right) g\left(\sigma\left(e_{i}, P e^{k}\right), F e^{j}\right)\right.} \\
& \left.-g\left(\sigma\left(e_{i}, e^{k}\right), F e^{j}\right) g\left(\sigma\left(\Psi e_{i}, P e^{k}\right), F e^{j}\right)\right] \\
= & -4 p \cos ^{2} \theta\|\nabla \ln \Phi\|^{2},
\end{aligned}
$$

where $\left\{e_{0}=\eta, e_{1}, e_{2}, \cdots, e_{q}, \Psi e_{1}, \Psi e_{2}, \cdots, \Psi e_{q}\right\}$ and $\left\{e^{1}, e^{2}\right.$, $\left.\cdots, e^{p}, e^{p+1}=\sec \theta P e^{1}, e^{p+2}=\sec \theta P e^{2}, \cdots, e^{2 p}=\sec \theta P e^{p}\right\}$ are the frames of the orthonormal vector fields on $T N_{T}$ and $T N_{\theta}$, respectively. 
Proof. First, we expand the left hand term in the following way:

$$
\begin{aligned}
& \sum_{i=1}^{q}\left[\sum_{j, k=1}^{2 p} g\left(\sigma\left(\Psi e_{i}, e^{k}\right), F e^{j}\right) g\left(\sigma\left(e_{i}, P e^{k}\right), F e^{j}\right)\right] \\
&=\sum_{i=1}^{q}\left[\sum_{j=1}^{2 p} g\left(\sigma\left(\Psi e_{i}, e^{j}\right), F e^{j}\right) g\left(\sigma\left(e_{i}, P e^{j}\right), F e^{j}\right)\right. \\
&+\left.\sum_{j \neq k=1}^{2 p} g\left(\sigma\left(\Psi e_{i}, e^{k}\right), F e^{j}\right) g\left(\sigma\left(e_{i}, P e^{k}\right), F e^{j}\right)\right] \\
&= \sum_{i=1}^{q}\left[\sum_{j=1}^{2 p} g\left(\sigma\left(\Psi e_{i}, e^{j}\right), F e^{j}\right) g\left(\sigma\left(e_{i}, P e^{j}\right), F e^{j}\right)\right. \\
&+\sum_{j=1}^{p} g\left(\sigma\left(\Psi e_{i}, e^{j}\right), F e^{j+p}\right) g\left(\sigma\left(e_{i}, P e^{j}\right), F e^{j+p}\right) \\
&\left.+\sum_{j=1}^{p} g\left(\sigma\left(\Psi e_{i}, e^{j+p}\right), F e^{j}\right) g\left(\sigma\left(e_{i}, P e^{j+p}\right), F e^{j}\right)\right] \\
&= \sum_{i=1}^{q}\left[\sum_{j=1}^{2 p} g\left(\sigma\left(\Psi e_{i}, e^{j}\right), F e^{j}\right) g\left(\sigma\left(e_{i}, P e^{j}\right), F e^{j}\right)\right. \\
&+\sum_{j=1}^{p e c} c^{2} \theta \sum_{j=1}^{p} g\left(\sigma\left(\Psi e_{i}, e^{j}\right), F P e^{j}\right) g\left(\sigma\left(e_{i}, P e^{j}\right), F P e^{j}\right)
\end{aligned}
$$

Using part (ii) of Lemma 6 and Lemma 7 and utilizing (28), we get

$$
\begin{aligned}
\sum_{i=1}^{q} & {\left[\sum_{j, k=1}^{2 p} g\left(\sigma\left(\Psi e_{i}, e^{k}\right), F e^{j}\right) g\left(\sigma\left(e_{i}, P e^{k}\right), F e^{j}\right)\right] } \\
& =\sum_{i=1}^{q}\left[-2 p \cos ^{2} \theta\left(e_{i} \ln \Phi\right)^{2}-2 p \cos ^{2} \theta\left(\Psi e_{i} \ln \Phi\right)^{2}\right] \\
& =-2 p \cos ^{2} \theta\|\nabla \ln \Phi\|^{2} .
\end{aligned}
$$

Replacing $e_{i}$ by $\Psi e_{i}$ in the above equation, we get

$$
\begin{aligned}
\sum_{i=1}^{q} & {\left[\sum_{j, k=1}^{2 p} g\left(\sigma\left(e_{i}, e^{k}\right), F e^{j}\right) g\left(\sigma\left(\Psi e_{i}, P e^{k}\right), F e^{j}\right)\right] } \\
& =2 p \cos ^{2} \theta\|\nabla \ln \Phi\|^{2} .
\end{aligned}
$$

By subtracting the above two findings, the required result gets attained.

The next theorem provides the following characterization.

Theorem 9. Suppose that $M=N_{T} \times{ }_{\psi} N_{\theta}$ is a WP-PSS submanifold of a Sasakian space form $\bar{M}(c)$ such that $N_{T}$ is a compact submanifold. Then, $M$ is a Riemannian product submanifold if the following inequalities hold

$$
\begin{gathered}
\sum_{i=1}^{2 q} \sum_{j=1}^{2 p}\left\|\sigma_{\mu}\left(e_{i}, e^{j}\right)\right\|^{2} \leq q p(c-1) \sin ^{2} \theta \\
-2 p\left(\cos ^{2} \theta+2 \cot ^{2} \theta\right)\|\nabla \ln \Phi\|^{2}, \\
\sum_{i=1}^{q} \sum_{j=1}^{2 p} g\left(\sigma_{\mu}\left(\Psi e_{i}, e^{j}\right), \sigma_{\mu}\left(e_{i}, P e^{j}\right)\right) \geq 0,
\end{gathered}
$$

where $\sigma_{\mu}$ stands for the component of $\sigma$ in $\mu$ and $(2 q+1)$ and $2 p$ are the dimensions of $N_{T}$ and $N_{\theta}$, respectively.

Proof. Let $Y \in T N_{T}$ and $V \in T N_{\theta}$ be unit vector fields. Then, by making use of (2), (6), and (22), we have

$$
\bar{R}(Y, \Psi Y, V, F V)=-\frac{c-1}{2} \sin ^{2} \theta\|Y\|^{2}\|V\|^{2} .
$$

However, the Codazzi equation gives

$$
\begin{aligned}
\bar{R}(Y, \Psi Y, V, F V)= & g\left(\nabla_{Y}^{\perp} \sigma(\Psi Y, V), F V\right) \\
& -g\left(\nabla_{\Psi Y}^{\perp} \sigma(Y, V), F V\right) \\
& +g\left(\sigma\left(Y, \nabla_{\Psi Y} V\right), F V\right) \\
& -g\left(\sigma\left(\Psi Y, \nabla_{Y} V\right), F V\right) \\
& -g\left(\sigma\left(\nabla_{Y} \Psi Y, V\right), F V\right) \\
& +g\left(\sigma\left(\nabla_{\Psi Y} Y, V\right), F V\right) .
\end{aligned}
$$

Now, we compute the values of the terms involved in (67). First, we get

$$
\begin{aligned}
g\left(\nabla_{Y}^{\perp} \sigma(\Psi Y, V), F V\right)= & Y g(\sigma(\Psi Y, V), F V) \\
& -g\left(\sigma(\Psi Y, V), \nabla_{Y}^{\perp} F V\right)
\end{aligned}
$$

Using part (ii) of Lemma 6 leads to

$$
\begin{aligned}
g\left(\nabla_{Y}^{\perp} \sigma(\Psi Y, V), F V\right)= & Y^{2} \ln \Phi\|V\|^{2}+2(Y \ln \Phi)^{2}\|V\|^{2} \\
& -g\left(\sigma(\Psi Y, V), \nabla_{Y}^{\perp} F V\right) .
\end{aligned}
$$
follows:

Now, we compute the last term of (69) using (11) as $g\left(\sigma(\Psi Y, V), \nabla_{Y}^{\perp} F V\right)=g\left(\sigma(\Psi Y, V), \bar{\nabla}_{Y}(\Psi V-P V)\right)$

By the use of (8) and (13), the above equation becomes

$$
\begin{aligned}
g\left(\sigma(\Psi Y, V), \nabla_{Y}^{\perp} F V\right)= & g\left(\sigma(\Psi Y, V),\left(\bar{\nabla}_{Y} \Psi\right) V+\Psi \bar{\nabla}_{Y} V\right) \\
& -g(\sigma(\Psi Y, V), \sigma(Y, P V))
\end{aligned}
$$


Using (4), (8), (26), and parts (ii) and (iii) of Lemma 6 , we get

$$
\begin{aligned}
g\left(\sigma(\Psi Y, V), \nabla_{Y}^{\perp} F V\right)= & (Y \ln \Phi)^{2}\left(1+\cos ^{2} \theta\right)\|V\|^{2}+\left\|\sigma_{\mu}(Y, V)\right\|^{2} \\
& -g(\sigma(\Psi Y, V), \sigma(Y, P V)) .
\end{aligned}
$$

Utilizing (72) in (69), we obtain

$$
\begin{aligned}
g\left(\nabla_{Y}^{\perp} \sigma(\Psi Y, V), F V\right)= & Y^{2} \ln \Phi\|V\|^{2} \\
& +(Y \ln \Phi)^{2} \sin ^{2} \theta\|V\|^{2} \\
& -\left\|\sigma_{\mu}(Y, V)\right\|^{2} \\
& +g(\sigma(\Psi Y, V), \sigma(Y, P V)) .
\end{aligned}
$$

Similarly, we can write

$$
\begin{aligned}
g\left(\nabla_{\Psi Y}^{\perp} \sigma(Y, V), F V\right)= & -(\Psi Y)^{2} \ln \Phi\|V\|^{2} \\
& -(\Psi Y \ln \Phi)^{2} \sin ^{2} \theta\|V\|^{2} \\
& +\left\|\sigma_{\mu}(\Psi Y, V)\right\|^{2} \\
& +g(\sigma(Y, V), \sigma(\Psi Y, P V)) .
\end{aligned}
$$

From the second part of Lemma 6, we get

$$
g\left(A_{F V} V, \Psi Y\right)=Y \ln \Phi\|V\|^{2} .
$$

Replacing $Y$ by $\nabla_{Y} Y$ (using the totally geodesicness of $\left.N_{T}, \nabla_{Y} Y \in T N_{T}\right)$ in the above equation, we have

$$
g\left(A_{F V} V, \Psi \nabla_{Y} Y\right)=\nabla_{Y} Y \ln \Phi\|V\|^{2} .
$$

By using (8), we obtain

$$
g\left(A_{F V} V, \Psi \bar{\nabla}_{Y} Y-\Psi \sigma(Y, Y)\right)=\nabla_{Y} Y \ln \Phi\|V\|^{2} .
$$

Since $N_{T}$ is totally geodesic in $M$, it can be ready to verify that $\sigma(Y, X) \in \mu$, for all $Y, X \in T N_{T}$. Using this and (13) in equation (77), we have

$$
g\left(\sigma\left(\nabla_{Y} \Psi Y, V\right), F V\right)=\nabla_{Y} Y \ln \Phi\|V\|^{2} .
$$

Similarly, we can write

$$
g\left(\sigma\left(\nabla_{\Psi Y} Y, V\right), F V\right)=-\nabla_{\Psi Y} \Psi Y \ln \Phi\|V\|^{2} .
$$

By use of (26) and the second part of Lemma 6, we attain

$$
\begin{aligned}
& g\left(\sigma\left(\Psi Y, \nabla_{Y} V\right), F V\right)=(Y \ln \Phi)^{2}\|V\|^{2}, \\
& g\left(\sigma\left(Y, \nabla_{\Psi Y} V\right), F V\right)=-(\Psi Y \ln \Phi)^{2}\|V\|^{2} .
\end{aligned}
$$

Substituting values from (66), (73), (74), (78), (79), (80), and (81) in (67) yields

$$
\begin{aligned}
-\frac{c-1}{2} \sin ^{2} \theta\|Y\|^{2}\|V\|^{2}= & Y^{2} \ln \Phi\|V\|^{2}+(\Psi Y)^{2} \ln \Phi\|V\|^{2} \\
& -(Y \ln \Phi)^{2} \cos ^{2} \theta\|V\|^{2} \\
& -(\Psi Y \ln \Phi)^{2} \cos ^{2} \theta\|V\|^{2} \\
& -\left\|\sigma_{\mu}(Y, V)\right\|^{2}-\left\|\sigma_{\mu}(\Psi Y, V)\right\|^{2} \\
& -\nabla_{Y} Y \ln \Phi\|V\|^{2}-\nabla_{\Psi Y} \Psi Y \ln \Phi\|V\|^{2} \\
& +g(\sigma(\Psi Y, V), \sigma(Y, P V)) \\
& -g(\sigma(Y, V), \sigma(\Psi Y, P V)) .
\end{aligned}
$$

Let $\left\{e_{0}=\eta, e_{1}, e_{2}, \cdots, e_{q}, e_{q+1}=\Psi e_{1}, e_{q+2}=\Psi e_{2}, \cdots, e_{2 q}=\right.$ $\left.\Psi e_{q}\right\}$ be the orthonormal frame on $T N_{T}$ and $\left\{e^{1}, e^{2}, \cdots\right.$, $\left.e^{p}, \sec \theta P e^{1}, \sec \theta P e^{2}, \cdots, \sec \theta P e^{p}\right\} \quad$ be an orthonormal frame on $T N_{\theta}$. By using the decomposition (40) and formulas (42), (8), and (4) in the last term of the above equation using the orthonormal frame of $T N_{\theta}$, we get

$$
\begin{aligned}
-\frac{c-1}{2} & \sin ^{2} \theta\|Y\|^{2}\|V\|^{2} \\
= & Y^{2} \ln \Phi\|V\|^{2}+(\Psi Y)^{2} \ln \Phi\|V\|^{2}-(Y \ln \Phi)^{2} \cos ^{2} \theta\|V\|^{2} \\
& -(\Psi Y \ln \Phi)^{2} \cos ^{2} \theta\|V\|^{2}-\left\|\sigma_{\mu}(Y, V)\right\|^{2} \\
& -\left\|\sigma_{\mu}(\Psi Y, V)\right\|^{2}-\nabla_{Y} Y \ln \Phi\|V\|^{2}-\nabla_{\Psi Y} \Psi Y \ln \Phi\|V\|^{2} \\
& +\csc ^{2} \theta \sum_{j=1}^{2 p}\left[g\left(\sigma(\Psi Y, V), F e^{j}\right) g\left(\sigma(Y, P V), F e^{j}\right)\right. \\
& \left.-g\left(\sigma(\Psi Y, V), F e^{j}\right) g\left(\sigma(\Psi Y, P V), F e^{j}\right)\right]\left\|e^{j}\right\|^{2} \\
& +2 g\left(\sigma_{\mu}(\Psi Y, V), \sigma_{\mu}(Y, P V)\right) .
\end{aligned}
$$

Now, taking the sum of the above equation over $i=1$, $2, \cdots, q$ and $j=1,2, \cdots 2 p$, using (28) and (29) and part (iii) of Lemma 6, one can get

$$
\begin{aligned}
2 p \Delta(\ln \Phi)= & q p(c-1) \sin ^{2} \theta-2 p \cos ^{2} \theta\|\nabla \ln \Phi\|^{2} \\
& -\sum_{i=1}^{2 q} \sum_{j=1}^{2 p}\left\|\sigma_{\mu}\left(e_{i}, e^{j}\right)\right\|^{2}-4 p \cot ^{2} \theta\|\nabla \ln \Phi\|^{2} \\
& +2 \sum_{i=1}^{q} \sum_{j=1}^{2 p} g\left(\sigma_{\mu}\left(\Psi e_{i}, e^{j}\right), \sigma_{\mu}\left(e_{i}, P e^{j}\right)\right) .
\end{aligned}
$$

From (84), if

$$
\begin{aligned}
& \sum_{i=1}^{2 q} \sum_{j=1}^{2 p}\left\|\sigma_{\mu}\left(e_{i}, e^{j}\right)\right\|^{2} \\
& \quad \leq q p(c+1) \sin ^{2} \theta-2 p\left(\cos ^{2} \theta+2 \cot ^{2} \theta\right)\|\nabla \ln \Phi\|^{2}, \\
& \sum_{i=1}^{q} \sum_{j=1}^{2 p} g\left(\sigma_{\mu}\left(\Psi e_{i}, e^{j}\right), \sigma_{\mu}\left(e_{i}, P e^{j}\right)\right) \geq 0,
\end{aligned}
$$


then $\Delta \ln \Phi \geq 0$; hence, from Hopf's Lemma, $\ln \Phi$ is constant which means that $\Phi$ is constant, which proves the theorem.

The squared norm of $\sigma$ with reference to the warping function and slant function is obtained in the next theorem as follows.

Theorem 10. Let $\bar{M}(c)$ be a $(2 m+1)$-dimensional Sasakian space form and $M=N_{T} \times{ }_{\Phi} N_{\theta}$ be an n-dimensional WPPSS submanifold such that $N_{T}$ is a $(2 q+1)$-dimensional invariant submanifold and $N_{\theta}$ is a $2 p$-dimensional proper pointwise slant submanifold of $\bar{M}(c)$. If

$$
\sum_{i=1}^{q} \sum_{j=1}^{2 p} g\left(\sigma\left(\Psi e_{i}, e^{j}\right), \sigma\left(e_{i}, P e^{j}\right)\right) \geq 0
$$

then

$$
\begin{aligned}
(i)\|\sigma\|^{2} \geq & q p(c-1) \sin ^{2} \theta+2 p \sin ^{2} \theta\|\nabla \ln \Phi\|^{2} \\
& -2 p \Delta(\ln \Phi) .
\end{aligned}
$$

(ii) the necessary and sufficient conditions for the equality sign of (87) to be held identically are

(a) $N_{T}$ is the totally geodesic invariant in $\bar{M}(c)$. Furthermore, it is a Sasakian space form

(b) $N_{\theta}$ is totally umbilical in $\bar{M}(c)$

(c) $\sum_{i=1}^{q} \sum_{j=1}^{2 p} g\left(\sigma\left(\Psi e_{i}, e^{j}\right), \sigma\left(e_{i}, P e^{j}\right)\right)=0$

Proof. From (84), we have

$$
\begin{aligned}
\sum_{i=1}^{2 q} \sum_{j=1}^{2 p}\left\|\sigma_{\mu}\left(e_{i}, e^{j}\right)\right\|^{2} \geq & q p(c-1) \sin ^{2} \theta-2 p\left(\cos ^{2} \theta\right. \\
& \left.+2 \cot ^{2} \theta\right)\|\nabla \ln \Phi\|^{2}-2 p \Delta(\ln \Phi)
\end{aligned}
$$

For the orthonormal frames $\left\{e_{0}=\eta, e_{1}, e_{2}, \cdots, e_{q}, e_{q+1}=\right.$ $\left.\Psi e_{1}, e_{q+2}=\Psi e_{2}, \cdots, e_{2 q}=\Psi e_{q}\right\}$ and $\left\{e^{1}, e^{2}, \cdots, e^{p}, \sec \theta P e^{1}\right.$, $\left.\sec \theta P e^{2}, \cdots, \sec \theta P e^{p}\right\}$, in view of formulas (40) and (41) and part (ii) of Lemma 6, we get

$$
\begin{aligned}
\sum_{i=0}^{2 q} \sum_{j=1}^{2 p}\left\|\sigma_{F D_{\theta}}\left(e_{i}, e^{j}\right)\right\|^{2} & =\sum_{i=0}^{2 q} \sum_{j, k=1}^{2 p} \csc ^{2} \theta g\left(\sigma\left(e_{i}, e^{j}\right), F e^{k}\right)^{2} \\
= & \csc ^{2} \theta \sum_{i=0}^{2 q}\left[\sum_{j=1}^{2 p} g\left(\sigma\left(e_{i}, e^{j}\right), F e^{j}\right)^{2}\right. \\
& \left.+\sum_{j \neq k=1}^{2 p} g\left(\sigma\left(e_{i}, e^{j}\right), F e^{k}\right)^{2}\right]
\end{aligned}
$$

$$
\begin{aligned}
& =\csc ^{2} \theta \sum_{i=0}^{2 q}\left[2 p\left(e_{i} \ln \Phi\right)^{2}\right. \\
& +\sec ^{2} \theta \sum_{j=1}^{p}\left\{g\left(\sigma\left(e_{i}, e^{j}\right), F P e^{j}\right)^{2}\right. \\
& \left.\left.+g\left(\sigma\left(e_{i}, P e^{j}\right), F e^{j}\right)^{2}\right\}\right] .
\end{aligned}
$$

Further using Lemma 7 and (28), the above equation is reduced to

$\sum_{i=0}^{2 q} \sum_{j=1}^{2 p}\left\|\sigma_{F D_{\theta}}\left(e_{i}, e^{j}\right)\right\|^{2}=2 p \csc ^{2} \theta\|\nabla \ln \Phi\|^{2}+2 p \cot ^{2} \theta\|\nabla \ln \Phi\|^{2}$.

From (88) and (90), we attain the desired inequality.

To prove part (ii), suppose that $\sigma^{\prime}$ is the second fundamental form for the immersion of $N_{\theta}$ in $M$. Then, $\forall V, U \in T N_{\theta}$ and $Y \in T N_{T}$, the Gauss formula gives

$g\left(\sigma^{\prime}(V, U), Y\right)=g\left(\nabla_{V} U, Y\right)=-Y \ln \Phi g(V, U)$.

Using (27), we attain

$$
\begin{gathered}
g\left(\sigma^{\prime}(V, U), Y\right)=-g(V, U) g(\nabla \ln \Phi, Y), \\
\quad \operatorname{or} \sigma^{\prime}(V, U)=-g(V, U) \nabla \ln \Phi
\end{gathered}
$$

For the equality case of (87), we get

$$
\begin{array}{r}
\sigma(D, D)=0, \sigma\left(D^{\theta}, D^{\theta}\right)=0, \\
g\left(\sigma_{\mu}\left(\Psi D, D^{\theta}\right), \sigma_{\mu}\left(D, P D^{\theta}\right)\right)=0 .
\end{array}
$$

From the first equation of (94), we go to the conclusion that $N_{T}$ is totally geodesic in $M$. Furthermore, one can readily check that $g(\sigma(Y, \Psi X), F V)=0, \forall Y$, $X \in T N_{T}, \quad V \in T N_{\theta}$. It leads to the conclusion that $N_{T}$ is totally geodesic in $\bar{M}(c)$ and it is a Sasakian space form as well. Moreover, the second equation of (94) together with (93) yields that $N_{\theta}$ is totally umbilical. This proves the theorem.

\section{Some Applications}

Theorem 10 motivates to compute the formula for Dirichlet energy for the warping function $\Phi$. Here, the Dirichlet energy of $\Phi$ is denoted by $E(\Phi)$ and is calculated for a compact orientable WP-PSS submanifold $M=N_{T} \times{ }_{\Phi} N_{\theta}$ in a Sasakian space form $\bar{M}(c)$ as follows.

Theorem 11. Suppose that $M=N_{T} \times{ }_{\Phi} N_{\theta}$ is a compact orientable WP-PSS submanifold of a Sasakian space form $\bar{M}(c)$, 
such that $N_{T}$ is a $(2 q+1)$-dimensional invariant submanifold tangent to the structure vector field $\eta$ and $N_{\theta}$ is a $2 p$ dimensional pointwise slant submanifold of $\bar{M}(c)$. Then, for every $y \in N_{\theta}$, the necessary and sufficient conditions for the Dirichlet energies of the warping functions to fulfil the following

$$
E(\ln \Phi)=\frac{1}{4 p \sin ^{2} \theta} \int_{N_{T} \times\{y\}}\|\sigma\|^{2} d V-\frac{p(c-1)}{4} \operatorname{Vol}\left(N_{T}\right)
$$

are the following:

(i) $N_{T}$ is the totally geodesic invariant in $\bar{M}(c)$, and it is a Sasakian space form

(ii) $N_{\theta}$ is totally umbilical in $\bar{M}(c)$

(iii) $\sum_{i=1}^{q} \sum_{j=1}^{2 p} g\left(\sigma\left(\Psi e_{i}, e^{j}\right), \sigma\left(e_{i}, P e^{j}\right)\right)=0$

Proof. By integrating the case of equality of (87) and using the definition of Dirichlet energy and (33), we get the desired result.

If $\theta=\pi / 2$, the compact orientable WP-PSS submanifolds become contact CR-warped product submanifolds. The above theorem can be used for deducing the following results.

Corollary 12. Suppose that $M=N_{T} \times{ }_{\Phi} N_{\perp}$ is a compact orientable contact CR-warped product submanifold of a Sasakian space form $\bar{M}(c)$, such that $N_{T}$ is a $(2 q+1)$ dimensional invariant submanifold tangent to $\eta$ and $N_{\perp}$ is a 2p-dimensional anti-invariant submanifold of $\bar{M}(c)$. Then, for every $x \in N_{\perp}$, the necessary and sufficient conditions for the relation

$$
E(\ln \Phi)=\frac{1}{4 p} \int_{N_{T} \times\{y\}}\|\sigma\|^{2} d V-\frac{p(c-1)}{4} \operatorname{Vol}\left(N_{T}\right)
$$

to be held are the following:

(i) $N_{T}$ is a Sasakian space form and totally geodesic invariant in $\bar{M}(c)$

(ii) $N_{\perp}$ is a totally umbilical anti-invariant in $\bar{M}(c)$

If equality sign of (87) holds, then

$$
\begin{aligned}
\sum_{i=1}^{2 q} \sum_{j=1}^{2 p}\left\|\sigma_{\mu}\left(e_{i}, e^{j}\right)\right\|^{2}= & q p(c-1) \sin ^{2} \theta-2 p\left(\csc ^{2} \theta\right. \\
& \left.+2 \cot ^{2} \theta\right)\|\nabla \ln \Phi\|^{2}-2 p \Delta \ln \Phi .
\end{aligned}
$$

The Laplacian of a smooth function $\Phi$ is the trace of the Hessian of the function. In terms of Hessian, (98) can be written as follows:

$$
\begin{aligned}
& \sum_{i=1}^{2 q} \sum_{j=1}^{2 p}\left\|\sigma_{\mu}\left(e_{i}, e^{j}\right)\right\|^{2}=q p(c-1) \sin ^{2} \theta-2 p\left(\csc ^{2} \theta\right. \\
& \left.\quad+2 \cot ^{2} \theta\right)\|\nabla \ln \Phi\|^{2}+2 p \sum_{i=1}^{q}\left[\operatorname{Hess}^{\ln \Phi}\left(e_{i}, e_{i}\right)\right. \\
& \left.\quad+\operatorname{Hess}^{\ln \Phi}\left(\Psi e_{i}, \Psi e_{i}\right)\right] .
\end{aligned}
$$

Now, the study comprises the following classification theorem for the WP-PSS submanifolds admitting the gradient Ricci soliton fulfilling the equality case of (87).

Theorem 13. Suppose that $\bar{M}(c)$ is a $2 m+1$-dimensional Sasakian space form and $M=N_{T} \times{ }_{\Phi} N_{\theta}$ is a WP-PSS submanifold admitting a shrinking gradient Ricci soliton. Then, if

$\sum_{i=1}^{2 q} \sum_{j=1}^{2 p}\left\|\sigma_{\mu}\left(e_{i}, e^{j}\right)\right\|^{2}+4 q p=q p(c-1) \sin ^{2} \theta+2 p \sum_{i=1}^{2 q} \operatorname{Ric}\left(e_{i}, e_{i}\right)$,

one among the following is true:

(i) $\theta=\pi / 2$, i.e., $M$ is a contact CR-warped product submanifold

(ii) $\Phi$ is constant, i.e., $M$ is trivial Riemannian product pointwise semi slant submanifold

Proof. Suppose that the WP-PSS submanifold $M=N_{T} \times{ }_{\psi} N_{\theta}$ satisfies the basic equation of the Ricci soliton, such that the potential function $\tau=\ln \Phi$, then

$$
\operatorname{Ric}(Y, X)=\alpha g(Y, X)+\operatorname{Hess}^{\tau}(Y, X),
$$

for all $Y, X \in T N_{T}$. Considering that $\left\{e_{1}, e_{2}, \cdots, e_{q}, e_{q+1}=\right.$ $\left.\Psi e_{1}, \cdots, e_{2 p}=\Psi e_{q}\right\}$ is an orthonormal basis of the vector fields on $T N_{T}$. Now, taking summation over $i=1,2, \cdots, q$ for $Y=X$ in (101), we have

$$
\sum_{i=1}^{q} \operatorname{Ric}\left(e_{i}, e_{i}\right)=\alpha q+\sum_{i=1}^{q} \operatorname{Hess}^{\tau}\left(e_{i}, e_{i}\right)
$$

Replacing $e_{i}$ by $\Psi e_{i}$ in the above equation, we get

$$
\sum_{i=1}^{q} \operatorname{Ric}\left(\Psi e_{i}, \Psi e_{i}\right)=\alpha q+\sum_{i=1}^{q} \operatorname{Hess}^{\tau}\left(\Psi e_{i}, \Psi e_{i}\right)
$$


From (102) and (103), we have

$$
\sum_{i=1}^{2 q} \operatorname{Ric}\left(e_{i}, e_{i}\right)=2 \alpha q++\sum_{i=1}^{q}\left(\operatorname{Hess}^{\tau}\left(e_{i}, e_{i}\right)+\operatorname{Hess}^{\tau}\left(\Psi e_{i}, \Psi e_{i}\right)\right)
$$

By the assumption that the equality in (87) holds, then by (99),

$$
\begin{aligned}
& \frac{1}{2 p} \sum_{i=1}^{2 q} \sum_{j=1}^{2 p}\left\|\sigma_{\mu}\left(e_{i}, e^{j}\right)\right\|^{2}=\frac{q(c-1)}{2} \sin ^{2} \theta \\
& \quad-\left(\cos ^{2} \theta+2 \cot ^{2} \theta\right)\|\nabla \ln \Phi\|^{2}+\sum_{i=1}^{2 q} \operatorname{Ric}\left(e_{i}, e_{i}\right)-2 \alpha q, \\
& \text { or } \sum_{i=1}^{2 q} \sum_{j=1}^{2 p}\left\|\sigma_{\mu}\left(e_{i}, e^{j}\right)\right\|^{2}=q p(c-1) \sin ^{2} \theta \\
& -2 p\left(\cos ^{2} \theta+2 \cot ^{2} \theta\right)\|\nabla \ln \Phi\|^{2}+2 p \sum_{i=1}^{2 q} \operatorname{Ric}\left(e_{i}, e_{i}\right)-4 q p \alpha .
\end{aligned}
$$

By assumption (100), we get

$$
\left(\cos ^{2} \theta+2 \cot ^{2} \theta\right)\|\nabla \ln \Phi\|^{2}=0 .
$$

From the above equation, it is evident that $\theta=\pi / 2$ or $\Phi$ is constant, which proves the theorem.

If the submanifold $M=N_{T} \times{ }_{\Phi} N_{\theta}$ admits the steady gradient Ricci soliton, then the following can be easily concluded from the above theorem.

Theorem 14. Suppose that $\bar{M}(c)$ is a $2 m+1$-dimensional Sasakian space form and $M=N_{T} \times{ }_{\Phi} N_{\theta}$ is a WP-PSS submanifold admitting a steady gradient Ricci soliton. If the relation

$$
\sum_{i=1}^{2 q} \sum_{j=1}^{2 p}\left\|\sigma_{\mu}\left(e_{i}, e^{j}\right)\right\|^{2}=q p(c-1) \sin ^{2} \theta+2 p \sum_{i=1}^{2 q} \operatorname{Ric}\left(e_{i}, e_{i}\right)
$$

holds, then one can be true from the following:

(i) $\theta=\pi / 2$, i.e., $M$ is a contact CR-warped product submanifold

(ii) $\Phi$ is constant, i.e., $M$ is trivial Riemannian product pointwise semi slant submanifold

In terms of Ricci curvature, the study constitutes the following classification.

Theorem 15. Suppose that $\bar{M}(c)$ is a $2 m+1$-dimensional Sasakian space form and $M=N_{T} \times{ }_{\Phi} N_{\theta}$ is a WP-PSS submanifold with the equality in (87) holds. If the following holds
$2 p \int_{M} \operatorname{Ric}(\nabla \ln \Phi,-) d V=q p(c-1) \sin ^{2} \theta-\sum_{i=1}^{2 q} \sum_{j=1}^{2 p}\left\|\sigma_{\mu}\left(e_{i}, e^{j}\right)\right\|^{2}$

then one among the listed statements must be correct:

(i) $\theta=\pi / 2$, i.e., $M$ is a contact CR-warped product submanifold

(ii) $\Phi$ is constant, i.e., $M$ is trivial Riemannian product pointwise semi slant submanifold

Proof. For a connection $\nabla$ on a smooth manifold $M$, there exists a tensor $R$ of type $(1,3)$ called the curvature tensor of the connection $\nabla$ given by

$$
\nabla_{U} \nabla_{V} Z-\nabla_{V} \nabla_{U} Z-\nabla_{[V, U]} Z=R(V, U) Z
$$

$\forall V, U, Z \in T M$.

For a warping function $\tau=\ln \Phi$, from (109), we obtain

$$
\nabla^{2} d(\tau)(U, V, Z)-\nabla^{2} d(\tau)(V, U, Z)=d(\tau) R(V, U) Z
$$

By the smoothness property of $\Phi$ on $N_{T}$ and $\nabla_{V U}^{2}=$ $\nabla_{V} \nabla_{U}-\nabla_{\nabla_{V} U}$, then $R(V, U) Z$ behaves like a derivative. Since $d \tau$ is closed, then it is ready to check that $\nabla^{2} d(\tau)$ $(V, U, Z)=\nabla^{2} d(\tau)(U, V, Z), \forall V, V, Z \in T N_{T}$. Now, for a local orthonormal basis $\left\{e_{1}, e_{2}, \cdots, e_{2 q}\right\}$ on $N_{T}$ and for a fixed point $t \in N_{T}$ such that $\nabla_{e_{i}}\left(e_{j}\right)(t)=0$, for $1 \leq i, j \leq$ $2 q+1$. If we specify $\nabla_{e_{i}}(V)(t)=0$, for any $V \in T N_{T}$, and taking trace with respect to $U$ and $Z$ in the following equation:

$$
\nabla^{2} d(\tau)(U, V, Z)=\nabla^{2} d(\tau)(V, U, Z)
$$

and utilizing (110), we have

$$
\sum_{i=1}^{q}\left(\nabla^{2} d(\tau)\right)\left(e_{i}, e_{j}, V\right)=-d(\Delta(\tau))(V)+\operatorname{Ric}(\nabla, V) .
$$

Further solving the left hand side of (112), it becomes

$$
\operatorname{div}\left(\operatorname{Hess}^{\tau}\right)(V)+d(\Delta(\tau))(V)=\operatorname{Ric}(\nabla \tau, V)
$$

the above equation can be written in the form of an operator as follows:

$$
\operatorname{div}\left(\operatorname{Hess}^{\tau}\right)+d(\Delta(\tau))=\operatorname{Ric}(\nabla \tau, \cdots)
$$

Since $M=N_{T} \times{ }_{\Phi} N_{\theta}$ is a compact orientable WP submanifold, then on integrating 


$$
\Delta(\tau)+\int_{M} \operatorname{div}\left(\operatorname{Hess}^{\tau}\right) d V=\int_{M} \operatorname{Ric}(\nabla \tau, \cdots) d V
$$

where $d V$ is the volume element.

Since $\Delta \Phi=-\operatorname{div}(\nabla \Phi)[26]$ and $\int_{M} \operatorname{div}(V) d V=0$ for any $V \in T N_{T}$. So, it is easy to conclude that $\int_{M} \operatorname{div}\left(\mathrm{Hess}^{\tau}\right)$ $d V=0$. Then,

$$
\Delta(\tau)=\int_{M} \operatorname{Ric}(\nabla \tau, \cdots) d V
$$

Utilizing the above equation in (98), we have

$$
\begin{aligned}
& \sum_{i=1}^{2 q} \sum_{j=1}^{2 p}\left\|\sigma_{\mu}\left(e_{i}, e^{j}\right)\right\|^{2}=q p(c-1) \sin ^{2} \theta \\
& \quad-2 p\left(\csc ^{2} \theta+2 \cot ^{2} \theta\right)\|\nabla \ln \Phi\|^{2}-2 p \int_{M} \operatorname{Ric}(\nabla \ln \Phi, \cdots) d V
\end{aligned}
$$

By assumption (108), we get

$$
\left(\cos ^{2} \theta+2 \cot ^{2} \theta\right)\|\nabla \ln \Phi\|^{2}=0 .
$$

From the above equation, it is evident that $\theta=\pi / 2$ or $\Phi$ is constant, which proves the theorem.

\section{Conclusion}

We obtain the inequalities characterizing the existence of WP-PSS submanifolds of Sasakian space forms via Hopf's Lemma. Furthermore, we gave an estimation of the squared norm of the second fundamental form with regard to the slant function and warping function. To strengthen our results, we provided an example of a WP-PSS submanifold in a Sasakian manifold. Moreover, some applications in the form of the Dirichlet energy of the warping function are derived. The results obtained may be helpful in further studies on the Dirichlet energy of smooth functions.

\section{Abbreviations}

WP: $\quad$ Warped product

WF: Warping function

AC: $\quad$ Almost contact

WP-PSS: Warped product pointwise semi slant submanifold.

\section{Data Availability}

No data were used.

\section{Conflicts of Interest}

Both the authors declare that they have no competing interests.

\section{Authors' Contributions}

Both of the authors worked equally on this paper.

\section{Acknowledgments}

The project was funded by the Deanship of Scientific Research (DSR), King Abdulaziz University, Jeddah, under grant no. D-139-662-1440. The authors, therefore, gratefully acknowledge the DSR technical and financial support.

\section{References}

[1] J. K. Beem, P. Ehrlich, and T. G. Powell, Warped Product Manifolds in Relativity, Selected studies, North-Holland, Amsterdam-New York, 1982.

[2] S. W. Hawkings and G. F. R. Ellis, The Large Scale Structure of Space-Time, Cambridge University Press, Cambridge, 1973.

[3] B. O'Neill, Semi-Riemannian Geometry with Application to Relativity, Academic Pres, 1983.

[4] R. L. Bishop and B. O’Neill, "Manifolds of negative curvature," Transactions of the American Mathematical Society, vol. 145, pp. 1-49, 1969.

[5] B. Y. Chen, "Geometry of warped product CR-submanifolds in Kaehler manifolds," Monatshefte für Mathematik, vol. 133, no. 3, pp. 177-195, 2001.

[6] B. Y. Chen, "Geometry of warped product CR-submanifolds in Kaehler manifolds II," Monatshefte für Mathematik, vol. 134, no. 2, pp. 103-119, 2001.

[7] I. Hasegawa and I. Mihai, "Contact CR-warped product submanifolds in Sasakian manifolds," Geometriae Dedicata, vol. 102, no. 1, pp. 143-150, 2003.

[8] F. R. al-Solamy and M. A. Khan, "Semi-slant warped product submanifolds of a Kenmotsu manifold," Mathematical Problems in Engineering, vol. 2012, Article ID 708191, 10 pages, 2012.

[9] M. Atçeken, "Warped product semi-slant submanifolds in Kenmotsu manifolds," Turkish Journal of Mathematics, vol. 34, pp. 425-433, 2010.

[10] V. A. Khan, K. A. Khan, and S. Uddin, "A note on warped product submanifolds of Kenmotsu manifolds," Mathematica Slovaca, vol. 61, no. 1, pp. 1-14, 2011.

[11] S. Uddin, V. A. Khan, and K. A. Khan, "Warped product submanifolds of a Kenmotsu manifolds," Turkish Journal of Mathematics, vol. 36, pp. 319-330, 2012.

[12] K. S. Park, "Pointwise slant and pointwise semi-slant submanifolds in almost contact metric manifolds," http://arxiv.org/abs/1410.5587v2.

[13] B. Sahin, "Warped product pointwise semi-slant submanifolds of Kähler manifolds," Portugaliae Mathematica, vol. 70, no. 3, pp. 251-268, 2013.

[14] M. A. Khan, "Warped product pointwise semi-slant submanifolds of the complex space forms," Rendiconti del Circolo Matematico di Palerno Series 2, pp. 1-13, 2019.

[15] I. Mihai and S. Uddin, "Warped product pointwise semi-slant submanifolds of Sasakian manifolds," 2018, http://arxiv.org/abs/1706.04305v2.

[16] A. H. Alkhalidi and A. Ali, "Classification of warped product submanifolds in Kenmotsu Space forms admitting gradient Ricci Solitons,” Mathematics, vol. 7, no. 2, p. 112, 2019.

[17] M. Crasmareanu and P. Laurian-Ioan, "Ricci solitons on CRsubmanifolds of maximal CR-dimension in complex 
projective space," Carpathian Journal of Mathematics, vol. 32, pp. 173-177, 2016.

[18] J. R. Kim, "Remarks on the warped product structure from the Hessian of a function," Mathematics, vol. 6, no. 12, p. 275, 2018.

[19] M. Lemes de Sousa and R. Pina, "Gradient Ricci solitons with structure of warped product," Results in Mathematics, vol. 71, no. 3-4, pp. 825-840, 2017.

[20] D. E. Blair, Contact Manifolds in Riemannian Geometry Lecture Notes in Math. 509, Springer-Verlag, Berlin, 1976.

[21] J. L. Cabrerizo, A. Carriazo, L. M. Fernandez, and M. Fernandez, "Slant submanifolds in Sasakian Manifolds," Glasgow Mathematical Journal, vol. 42, no. 1, pp. 125-138, 2000.

[22] F. Etayo, "On quasi-slant submanifolds of an almost Hermitian manifold," Publicationes Mathematicae Debrecen, vol. 53, pp. 217-233, 1998.

[23] B. Y. Chen and O. Garay, "Pointwise slant submanifolds in almost Hermitian manifolds," Turkish Journal of Mathematics, vol. 36, pp. 630-640, 2012.

[24] S. Uddin and A. H. Al-Khalidi, "Pointwise slant submanifolds and their warped products in Sasakian manifolds," Filomat, vol. 32, no. 12, pp. 1-12, 2018.

[25] B. Y. Chen, "Pseudo-Riemannian Geometry, $\delta$-invariants and applications," World Scientific Publishing Company, Singapore, 2011.

[26] R. S. Hamilton, "Three-manifolds with positive Ricci curvature," Journal of Differential Geometry, vol. 17, no. 2, pp. 255-306, 1982. 


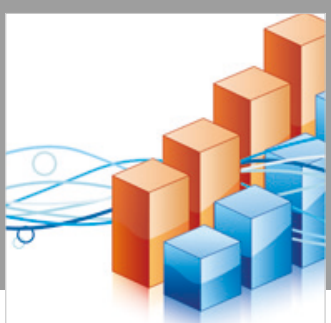

Advances in

Operations Research

\section{-n-m}
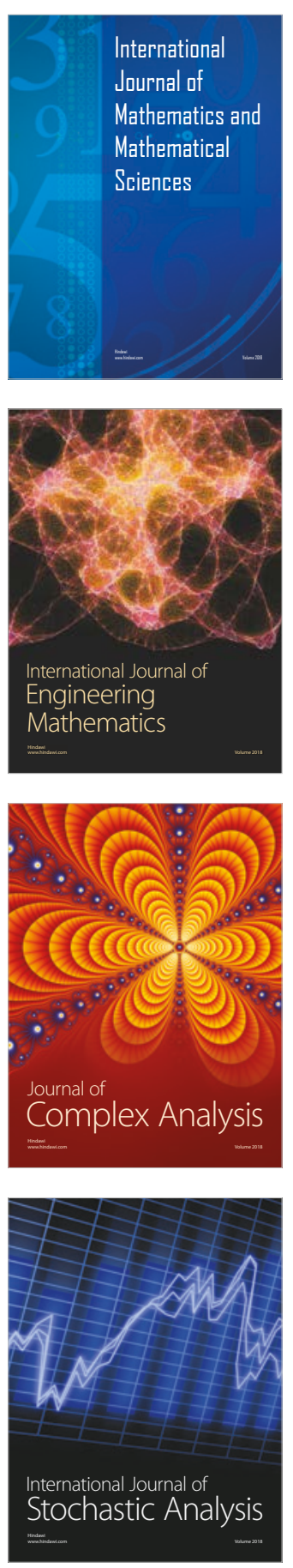
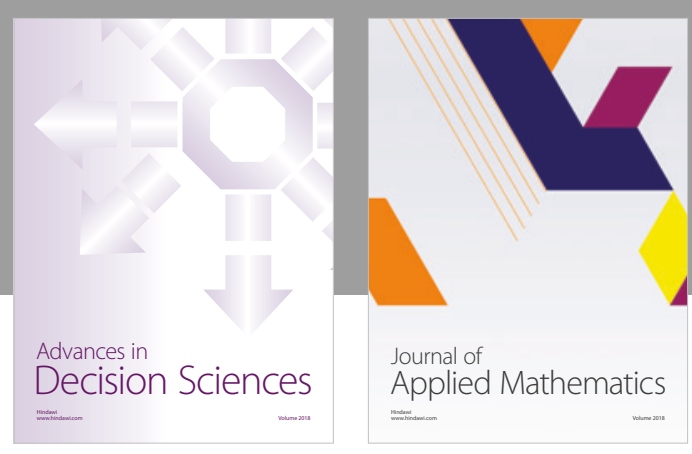

Journal of

Applied Mathematics
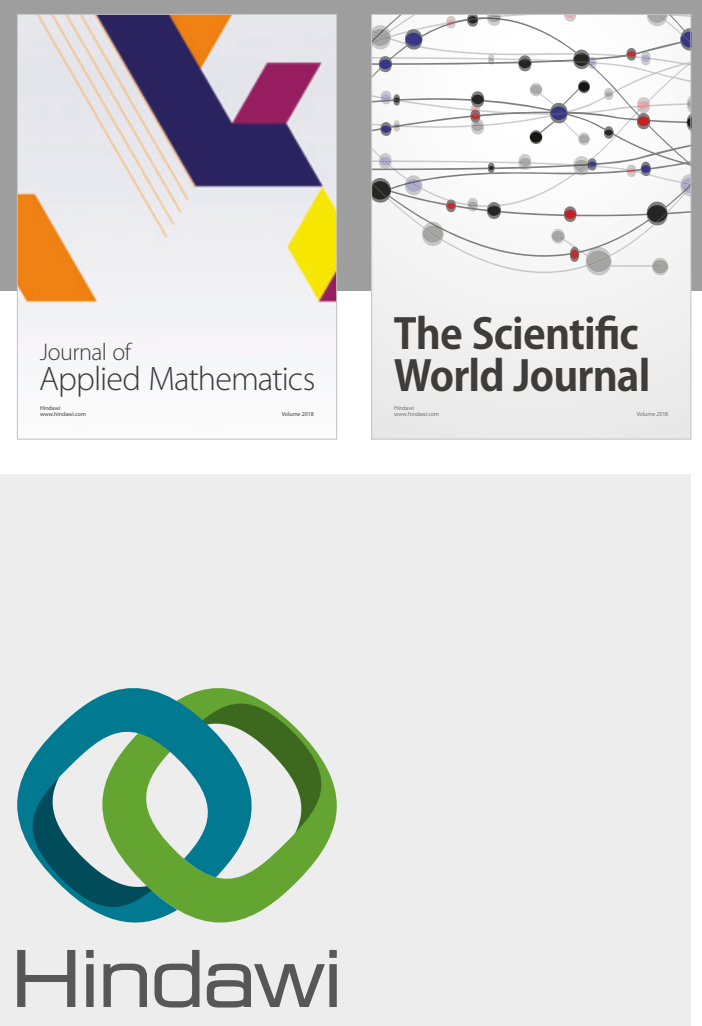

Submit your manuscripts at

www.hindawi.com

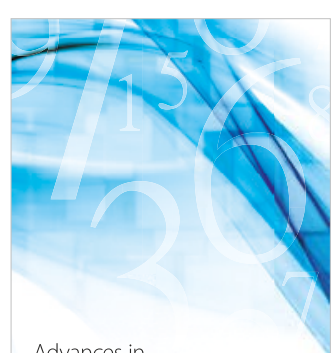

Advances in
Numerical Analysis
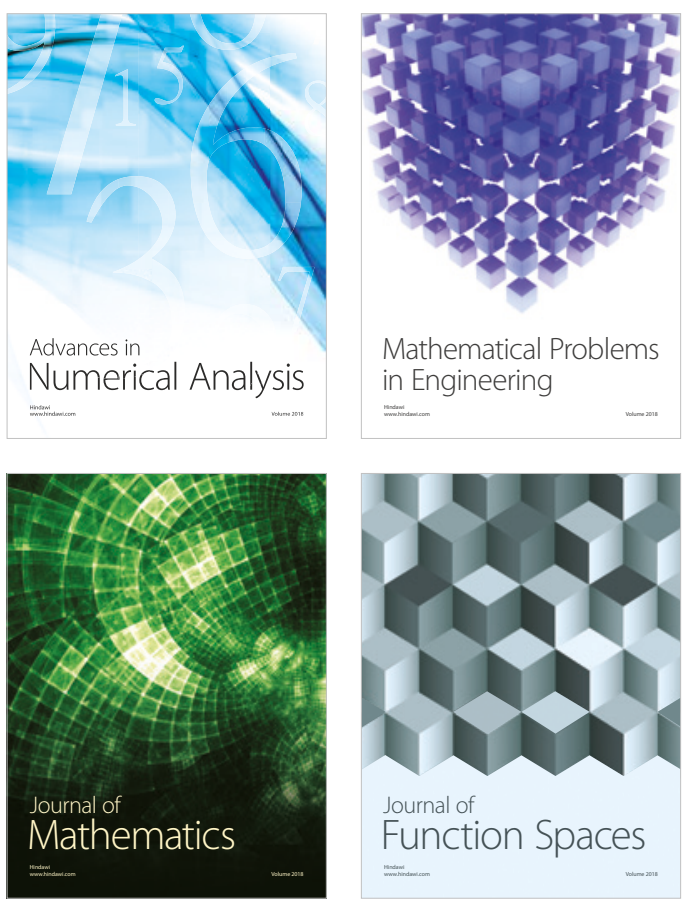

Mathematical Problems in Engineering

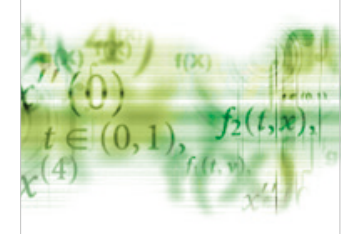

International Journal of

Differential Equations

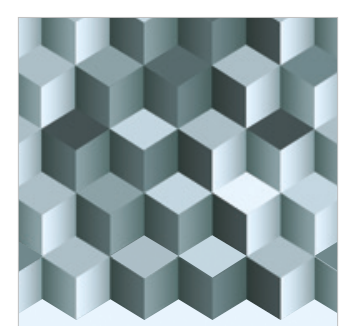

Journal of

Function Spaces

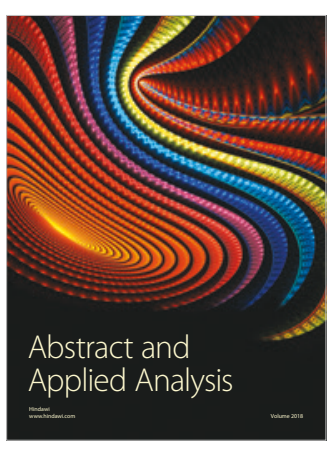

The Scientific

World Journal

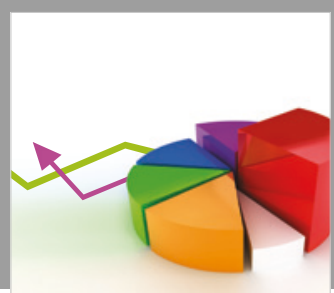

Journal of

Probability and Statistics
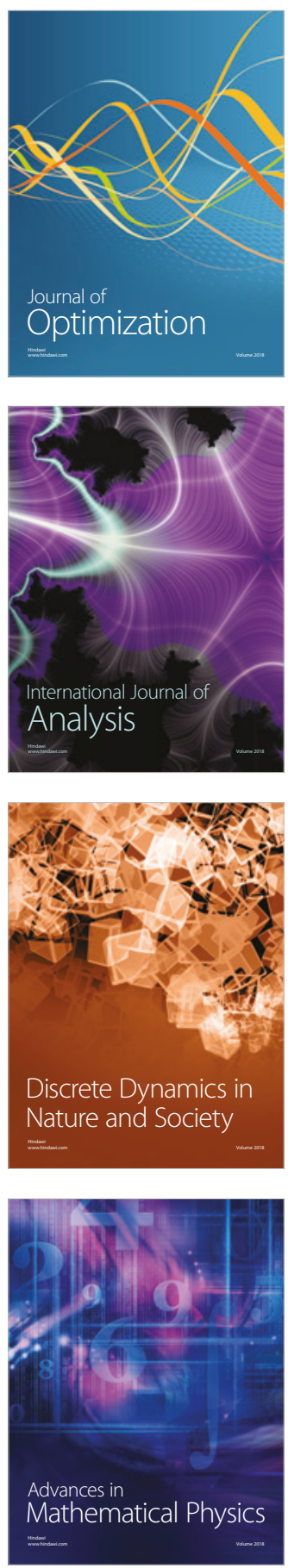